\title{
Concept Paper \\ Cultural Competence in Healthcare Leadership Education and Development
}

\author{
Steve Gulati * ${ }^{*}$ and Catherine Weir
}

check for

updates

Citation: Gulati, S.; Weir, C. Cultural Competence in Healthcare Leadership Education and Development. Societies 2022, 12, 39. https://doi.org/10.3390/soc12020039

Academic Editors: Costas S Constantinou, Panayiota Andreou, Monica Nikitara and Alexia Papageorgiou

Received: 21 December 2021 Accepted: 22 February 2022 Published: 2 March 2022

Publisher's Note: MDPI stays neutral with regard to jurisdictional claims in published maps and institutional affiliations.

Copyright: (C) 2022 by the authors. Licensee MDPI, Basel, Switzerland. This article is an open access article distributed under the terms and conditions of the Creative Commons Attribution (CC BY) license (https:// creativecommons.org/licenses/by/ $4.0 /)$.
Health Services Management Centre, College of Social Sciences, University of Birmingham, Birmingham B15 2RT, UK; c.s.weir@bham.ac.uk

* Correspondence: s.gulati@bham.ac.uk

\begin{abstract}
Cultural competence is a phenomenon that straddles many disciplines and fields of study There is no settled definition of the term, and it is argued that this is not necessary to explore or discuss the phenomenon as it is context-dependent across diverse societies. Explorations of cultural competence in clinical education and training are well-established, but there has been less attention towards its expression in the field of developing healthcare leaders. There is a debate about whether cultural competence is best achieved primarily through training-based educational inputs or by being infused in all areas of curriculum development. Using an exploration of selected literature followed by the case of an ambitious set of leadership development programmes in the English National Health Service, this paper explores the balance and interdependencies of cultural competence in healthcare leadership development as knowledge, skills and attitudes. The paper concludes that it is important for educators in this field to provide space for reflection, develop skills of reflexivity and facilitate sensitive discussions of sometimes contested ideas and concepts. A further evaluation of the impact of teaching and learning interventions, while mapping developments in perceptions of knowledge, skill and attitudes would be an area ripe for future research.
\end{abstract}

Keywords: leadership; healthcare leadership; cultural competence; leadership development; healthcare education; curriculum; equality; diversity and inclusion; NHS

\section{Introduction}

As the societies of many developed nations become increasingly complex and diverse, the concept of cultural competence across a range of disciplines in social science attracts growing amounts of attention. Health systems have long been challenged to address the needs of diverse and stratified societies, and cultural competence has tended to focus on the planning, commissioning and delivery of health services. This has often relied on post hoc interventions, sometimes designed to remedy an identified deficit, risk or shortcoming, at other times anticipatory or developmental. Cultural competence in the design and delivery of education and development, especially leadership development, is a relatively new concept, especially when this goes beyond traditional training. The purpose of this paper is to develop a more informed and nuanced view of the application of cultural competence to leadership development curricula, recognising that, historically, the focus has been on leveraging cultural competence interventions to reduce health inequalities and improve access to services. The concept of this paper is to explore the phenomenon of cultural competence through the lens of multidisciplinary healthcare leadership development interventions. To achieve this, we will examine cultural competence as a designed construct, that is, when it is part of the conceptual design rather than being added to an existing system, with a particular focus on the expression of cultural competence in educational and developmental models for healthcare professionals in the field of leadership development.

The debate will commence with a brief but broad mapping of the context within which the topic is located, before moving on to review some theoretical concepts and practical 
applications. The paper will then focus on the extent to which, and how, cultural competence was expressed in the pedagogic design of a suite of flagship leadership development programmes for healthcare leaders in the English National Health Service (NHS), before making some concluding comments about where cultural competence lies today as health systems move into the post-COVID-19-pandemic environment. Areas for potential further research will be identified.

\section{Cultural Competence in Healthcare Leadership Development-Framing the Debate}

Developing inclusive forms of leadership in healthcare can bring many potential benefits: emotional bonding and psychological engagement, and improving motivation, team effectiveness and innovation [1]. There is also the moral case of developing organisations that capture the benefits of a multiplicity of cultures and better represent the societies in which healthcare organisations are embedded, thereby seeking to improve patient experience and outcomes. The knowledge base upon which to seek to build inclusive workplaces in healthcare, however, is both congested and, to a degree, contested. From a leadership development perspective, what position does cultural competence take in the continuum of attending to issues at an individual as opposed to the systemic level?

\section{Concepts, Paradigms and Definitions}

In order to facilitate an informed debate, it important to firstly consider the terms used in this area of discussion. The foremost point to emerge here is that, although the concept of cultural competence in healthcare features increasingly frequently in policy and provision, the meaning and practice has been, and remains, contested [2]. Indeed, Sue et al., quoted in Horvat [3], argued that a prominent problem with the term was that "(a) it has various meanings, (b) includes inadequate descriptors, (c) is not theoretically grounded, and (d) is restricted by a lack of measurements and research designs for evaluating its impact in treatment". With emergent phenomena, however, or with phenomena that develop with time, it is arguably reductive to seek one single 'definition' [4]; a richer picture can be discerned through an exploratory and discursive approach, looking at how cultural competence has been discussed through various healthcare-related lenses, in order to develop a more informed and nuanced insight into the application of cultural competence to leadership development curricula, which is rooted in and aimed towards those working in healthcare. This notwithstanding, it is important to explore how cultural competency is framed in the field of healthcare more generally, and then in healthcare education. At its most basic, some studies posit this as no more than the provision of information, the provision of language skills, or the use of interpreters; we would suggest that, whilst this has merit in terms of developing operational 'toolkits' or similar tools, it does not propel the concept when exploring cultural competence in a more strategic sense, such as through curriculum design, a point which we will return to later. Deeper analyses frame this as a wider cultural change process that includes a more radical reimagination of the role of healthcare in communities, healthcare education and leadership development [5].

Considering this through the lens of the English National Health Service (NHS) the terminology of cultural competence, or at least its use as a relatively broad descriptor, has been prevalent for a number of years [6], although the practical expression of this for either employees or service users is harder to identify. This is in line with the wider literature. In more recent times, the language around cultural competence has been linked with that of equality, diversity and inclusion (EDI), as well as debates around power. This discourse is clearly linked to, but also distinct from, studies of health inequalities, determinants of health, and racial prejudice and bias. It is also important to consider some of the adjuncts to the concept, such as intercultural competence, cultural sensitivity, cultural humility or cultural intelligence [7], for again "while these terms are used interchangeably by some, each represents a different approach when working across cultural groups" [8] (p. 834), [9]. Each of these aspects has its own definitions and debates, with all adding to both the complexity and richness around these issues. Of particular relevance to this paper are 
the links to the domains of healthcare, where "cultural competence and cultural humility have been recommended as approaches to work with and serve diverse populations to address health disparities and increase health equity" [8] (p. 840); as well as connections to leadership, with the notion that intercultural competence can help leaders to overcome ethnocentrism [10] and generally improve leadership practice [11,12]. These ideas will run throughout this paper.

Having briefly set the scene, this paper will now proceed to explore cultural competence in the development of educational and developmental models for healthcare professionals in the field of leadership development, looking first at some theoretical perspectives before moving on to consider these as training interventions and focus on curricular applications.

\section{Theoretical Concepts}

Theoretical concepts around cultural competence in healthcare and healthcare leadership development arguably interweave with studies of migration, health determinants and health inequalities, racism and inclusion. This makes for an eclectic but rich area for study. Some of the debate around definitions has been explored above, but where might we look for theoretical underpinnings that can inform or focus this debate?

If wider socio-economic forces have brought the notion of cultural competence as a phenomenon to prominence, then it follows that a definition of it, whether narrow or more broadly encompassed, must in itself also be diverse; and as already mentioned, we argue that it is reductive to seek to settle on a single or specific definition. Garneau and Pepin [13] are eloquent about this in a piece that, whilst scoping a 'definition', spends considerable time exploring what underpins the concept and phenomenon of cultural competence (in this case through the lens of nursing), and by so doing raises a number of points worthy of consideration. They also draw an important read-across between culturally competent healthcare (in this case, nursing) practitioners and wider notions of cultural safety in the delivery and receipt of direct clinical services. Whilst the detailed concepts around patient, clinical and cultural safety are beyond the scope of this paper, this could be an area ripe for further exploration or primary research. In the social sciences concept of culture, especially when applied to organisations as organisms or as systems [14], attention tends to focus on social constructivism, in essence as a dynamic relational process that is both formed and affected by the actors involved. It is also worth noting studies around trust, which frame trust at different levels-individual relations, but also trust at organisational, systems and societal levels. In this regard, culturally competent pockets in society and organisations have limited efficacy, and can be viewed as iterative, organic processes. Kelly et al. [15] (p. 78) concur, concluding that "... most of the conceptual frameworks emphasise that cultural competence is an ongoing and evolving process, and that a lifelong commitment to self-reflection and continued education is essential if one is to become culturally competent". The importance of reflection, and indeed of reflexivity, will form a central plank of the analysis to follow, when considering both the curriculum and reviewing cultural competence in the design of a healthcare leadership development programme.

The theoretical or conceptual foundations also incorporate the role and position of the healthcare professional in society. For the purposes of this paper, 'healthcare professional' is interpreted widely and is not exclusive to clinicians or those providing direct healthcare, as it is the full architecture of the healthcare environment that impacts on patient or service user experience, and it is the entirety which needs to demonstrate cultural competence, irrespective of how this is framed. This can be neatly expressed by the assertion that the healthcare professional cannot be separated from either the system in which they work or the society in which the healthcare system is situated [13] (p. 13): "The professional must reflect and expand her vision of the power structures that can influence the social representations of the care, health, and culture. She could also reflect on the impact of these representations on individuals and on society". This argument finds its natural conclusion 
as set out by Markey and Oakantey [16] (p. 155), who make a plain connection between the principles of the effective and ethical delivery of healthcare services and those of cultural competence: "providing opportunities to apply the theoretical principles underpinning care to practice in a supportive environment is pivotal to developing cultural competence". Given the myriad of ideas, concepts and disciplines that combine to compose cultural competence, the very concept exists at the intersections of different aspects of the concept, with this intersectionality orbited by discussions of intercultural competence and cross, and inter-cultural, sensitivity, as mentioned above. Other areas of theoretical relevance are around group dynamics and intergroup contact theory [17], especially when considering the interplay between intercultural curiosity in diverse communities, both at societal and system (organisational) levels. A counterpoint to this is what could be termed 'cultural blindness', which represents some of the earlier thinking around 'equal opportunities' (in the United Kingdom at least). This posited that if a service is open to all and delivered in the same way, it is by definition 'fair', or that the healthcare system put in place by the dominant culture should work equally well for all cultures [18]. Even a cursory knowledge of the concept of diversity explodes this myth.

Therefore, as we can see, the theoretical framework around cultural competence in healthcare is broad, and reflective of many of the epistemological positions familiar to those engaged in the social sciences. It is important to consider this when taking into account the more specific issues around cultural competence in healthcare leadership development. This also leads to another key issue, which we will explore next: how these matters are reflected, contained and managed in healthcare related curricula.

\section{Application to Curriculum Development}

This section will explore how cultural competence has been expressed in the development of the curriculum in healthcare. A broad lens will be adopted, looking at both general applications and profession-specific factors, with a view to informing the later analysis of a particular leadership development approach in the English NHS. This section will open with a wider debate, before moving on to examine the specific issue of cultural competence as training interventions, which in itself serves to add to the debate around the purpose, aims and utility in designing culturally competent healthcare leadership development.

\subsection{Cultural Competence in Curriculum Development}

The manner in which cultural competence is framed and expressed through a healthcare provision and teaching lens can be informative in indicating purpose, meaning and execution ('why are we doing this', 'what it is', and 'is it working'). At a fundamental level, when developing a curriculum (or in fact training or awareness sessions), a common operating assumption is that a relatively simple educational input, based on teaching others about the differences in culture and encouraging tolerance and respect, is required [13]. Of course, on one level, there is merit and value in knowledge development and transfer, but as has been argued, this is likely to be insufficient in itself [13] for bringing about deeper, second-order insights, which are more likely to encouraged sustained behavioural change. There is also a critique that cultural competence initiatives focus on surface level matters such as, for instance, language barriers, translation services and the more obvious differences in race and ethnicities [9]; this could be conceived as a spectrum of interventions, with (perceived) 'facts' and 'how to guides' at one end, and much more complex, nuanced explorations and analyses of meaning and socio-economic-political determinants at the other [3]. Soule expresses this bluntly: "the traditional ways of conceptualising, teaching and learning cultural competence as a finite body of knowledge are both superficial and inadequate for the sweeping social and demographic changes occurring today" [19] (p. 196). This presents an interesting challenge when considering cultural competence in healthcare leadership development curricula-what assumptions are present, both dominant and passive, when framing the challenge? Whose views dominate, at both an individual and group (collective) level? Are these issues even visible, let alone explored? Whether, and 
the extent to which, these issues are ventilated at the conceptualisation and design stage of curriculum development are highly relevant to this discussion.

A significant portion of the literature explores how cultural competence is framed in nursing, medical or other uni-disciplinary professions, but contains less focus on the subject of this paper, multi-disciplinary leadership development programmes. Nevertheless, this can be instructive. In general terms, it is observed that "training curricula for medical, nursing and social work students now generally include lectures and coursework on cultural competency in healthcare provision" [2] (p. 7), but again familiar questions of definition or meaning arise, for the "... conceptualisation and implementation of cultural competence are poorly understood among healthcare practitioners and providers due to a lack of clarity in its definition... despite existing definitions incorporating similar terms, there remains a lack of conceptual clarity around the concept of cultural competence as the literature on the development of cultural competence is still evolving" [18] (p. 571). Furthermore, in their 2014 Cochrane Review, Horvat et al. [3] found that "... all studies used terminology and concepts such as cultural competence... or intercultural communication... but no consistent concept was used across the studies, nor did any study provide an explicit definition of the concept or terminology used" (p. 84). This notwithstanding, the literature is rich in examinations and analyses of medical and nursing education and training curricula with reference to cultural competence, and some commonalities in the discussion stand out $[2,5,6,20-25]$ :

o A curriculum-led approach to design in cultural competence in medical and nurse training is increasingly common, but still suffers from debates around definitions;

o The links between the determinants of health and culturally competent healthcare systems are less well-explored, with preference instead given to knowledge transfer of the (seemingly) objective, rather than subjective, aspects of cultural difference;

o Connections between personal and professional values, professional ethics and compassion in healthcare delivery feature in the drive to be culturally competent in some curriculum designs;

o The interplay between cultural competence, diversity, power and inclusion in curricula is an emerging and fluid field.

We will return to some of these points in Section 6. Additionally, one further point stands out from the literature when considering the concepts and impact of culturally competent healthcare- the balance between a strategic (curriculum- and attitudinal-based) and an operational (knowledge- and behaviour-based) approach. At a systemic level, it appears self-evident that working with one part of the environment has implications elsewhere, and that this will be compounded in complex, organic systems such as healthcare. Working on cultural awareness, issues of diversity and power with doctors and nurses is clearly important, but patient contacts are not limited to doctors and nurses, and the assumptions, knowledge and attitudes of all other professional groups, as well as those of support staff, are equally important $[21,26]$. This then becomes an issue of policy, organisational development and leadership, and it is this aspect, of whole systems and healthcare containing a highly diverse workforce in itself, which underpin the discussion in Section 6.

\subsection{Cultural Competence through Training}

Having explored some of the debates around a curricular approach to cultural competence for healthcare professionals, we will now focus further on the relationship between a curricular approach and cultural competence 'training' for individuals or groups. Clearly, the two are not in opposition and are in fact linked, but the balance between the two approaches in health systems can be revealing in terms of identifying underpinning motivating forces, and strategic thinking and intent. On one level, this speaks to critical stance and relates back to the earlier point about how the very concept is viewed, that is, as a 'deficit' that needs to be addressed (at an operational and practical level) or as a wider expression of privilege, power and inequality (at a more strategic and philosophical level). 
Before moving on to explore a case in the English NHS, some of the key aspects of this debate will be briefly explored.

On one level, it has been observed that "over the last two decades, cultural competence has become a more comprehensive, skill-based concept that involves the system... and has been conceived of as an ongoing quality improvement process, relevant across individual, organisational, systemic and professional levels" [3] (p. 7). Indeed, part of the discussion around (inter)cultural sensitivity is that it has both a cognitive and affective component [18], and arguably, this presents a challenge in the healthcare leadership development field and connects to the tension between facilitating development and 'telling' in a more didactic sense. This is similar with concepts of cultural intelligence, which span the cognitive, motivational and behavioural arenas [27]. The curriculum for a development programme can of course mandate aspects of cultural competence in the design, but it is individuals and groups who will interact with that curriculum, and the extent to which these participants have either the cognitive or affective aspects of cultural sensitivity, or indeed curiosity about it, is impossible to determine at the outset. This again puts the onus on the educational facilitative skill of programme tutors. At a conceptual level一and this applies to both curricula- or training-led approaches-it is important to recognise that both approaches are inevitably and fundamentally anchored in society. Whatever prejudices, stereotypes, myths, preferences or dislikes exist in any given society or social grouping will inevitably flavour its discourse $[2,20,21,28]$, and interventions fall into the broad categories of the longstanding training methodology of knowledge, skills and behavioural application of those skills, and awareness/attitudes. In practical terms, these tend to include traditional lectures to impart information about societal composition and demographics; case studies; case discussions; and role plays. There is also evidence $[2,3,16]$ from hearing the stories of minority or marginalized groups (usually patients) first-hand, and from the inclusion of time and space for reflection and reflective practices, both at individual and group levels. These are all, to a greater or lesser extent, traditional training methods.

A final point with regard to training is the practice, increasingly common in the healthcare field, of compulsory or mandatory training (often badged as equality and diversity training, sometimes as power and inclusion). Although the motivation behind such compulsion may be worthy, this approach arguably moves away from being rooted in matters of values, ethics or even morals $[18,29,30]$, and more towards a compliance-based practice. There is clearly a tension between mandating education or training interventions around cultural competence, because they are important, against the notion that cultural competence in a healthcare workforce is as much about attitudes and values than dry knowledge alone. Compulsory mandates to attend cultural competence training programmes can lead to resistance [20] or at best superficial participation, possibly invoking sensitivities by suggesting that the performance of individuals is somehow sub-optimal; it is counterproductive to 'other' the 'culturally incompetent' [31], with one of the risks of so doing creating an active disengagement or tokenistic expressions of compliance [32]. Nevertheless, it has been noted that professional bodies and regulators increasingly respond to the prevailing environment of diversity by "... mandating cultural competence training for healthcare professionals and healthcare education" [19] (p. 48) Whilst some aspects of diversity can realistically lend themselves to knowledge-transfer and training, the notion that cultural competence in healthcare is something that can be 'trained into' people needs to be handled with caution, and training interventions which are not rooted in a wider organisational development strategy are less likely to have a long-term impact.

\section{Cultural Competence in Healthcare Leadership Development-A Case Study}

Having explored some of the relevant theoretical concepts, this paper will now proceed to explore whether, and if so how, cultural competence was approached in the design of the largest suite of leadership development programmes in the history of the English National Health Service. Using publicly available sources and material, this section will describe the various leadership development offers made by the NHS Leadership Academy (NHSLA), 
before moving on to a discussion about what this might indicate about the development and use of cultural competence in the multi-disciplinary leadership development for healthcare workers.

The NHS Leadership Academy (NHSLA) was established in the English NHS in the wake of the Francis Inquiry (and subsequent Francis Report), which identified how skewed leadership priorities and behaviours had led to increased deaths and suffering in an acute hospital in the English Midlands [33]. A primary purpose of the NHSLA was to improve the quality of leadership in the NHS, doing so through the development of individuals and groups located in systems. Of the NHSLA suite of leadership development programmes, the Elizabeth Garrett Anderson Programme (henceforth the 'Anderson Programme') is the largest: a part time, two-year programme that was awarded Gold for Excellence in Practice by the European Federation of Management Development in 2016. Successful completion of the programme leads to an NHS Leadership Academy Award in Senior Healthcare Leadership and a master's degree in healthcare leadership. The numbers are significant; over 3500 people have participated in the Anderson programme, with nearly $50 \%$ of recent graduates reporting being promoted during their time on the programme, and $90 \%$ attributing this directly to their learning. The master's degree element of the programme is delivered through two universities. Participation in the programme is through competitive application, with the target demographic being aspiring leaders from both clinical and non-clinical backgrounds, who then go on to learn in multi-disciplinary groups; all participants work in the NHS. The marketing materials for the programme identify the programme as being for those:

o Aspiring to take on a more senior leadership role as well as looking to have a wider impact by leading a culture of compassion;

o Ready and committed to developing their leadership skills and behaviours, whilst undertaking a healthcare-related academic qualification;

o Motivated by the opportunity to apply new skills, learning and behaviours directly to real-time work-related improvements.

It is interesting to note that, in publicly available material on the programme, there are no references to cultural competence, although there is considerable emphasis on inclusion, equality and diversity. It is also of interest to note that a 2018 independent study, commissioned by the NHSLA to review the demand for director level leadership development across health and care, also made no mention of cultural competence. Instead, the 429 directors who responded identified the following top three development needs: (i) systems leadership (60 per cent identified this); (ii) leading without authority through others (38 per cent); and (iii) resilience ( 25 per cent).

The NHSLA programmes are underpinned by four key leadership principles [34], which are used purposefully throughout the programme. These are:

o Making person-centred co-ordinated care happen;

o Creating a culture for quality;

o Improving the quality of patient experience;

o Understanding oneself to improve the quality of care.

The principles express the values of the programme and its intention to improve leadership and management practice through focusing on equality, diversity and inclusion. These aims run through the whole suite of programmes developed by the NHSLA for NHS staff. Of these programmes, two are specifically targeted at addressing the current underrepresentation of ethnic-minority staff in leadership positions in the NHS. The Stepping Up programme [35] is a leadership development programme for ethnic minority staff within the NHS (termed 'BAME': Black, Asian and minority ethnic, although this term is now the subject of some debate), which aims to "create sustainable inclusion within the NHS by addressing the social, organisational and psychological barriers restricting BAME staff from progressing". The Ready Now programme [35] is advertised as a positive action programme for BAME senior leaders underpinned by a recognition that wide system change is required 
if inclusion is to be "lived, felt and sustained" and this programme is positioned as part of the wider work on inclusion and addressing "power imbalances... to embrace a more diverse talent pipeline". Some of the comments resulting from the evaluation of Ready Now [35] included "it provides a safe environment to explore the impact of leadership on underrepresented groups in the NHS", and "my engagements with others are viewed more critically through the lens of inclusiveness for patient benefit, staff benefit and organisational benefit. My actions are informed by a social inclusion agenda when considering design of services, service development and in staff management and engagement across all levels of the organisation".

In 2019, the NHSLA launched 'Building Leadership for Inclusion' (BLFI) [36], which aimed to expand the knowledge base around equality and diversity, with a particular focus on systemic and cultural change through leadership development. The stated the intention of BLFI was to ensure those with lived experience of systemic discrimination were able to contribute to reshaping leadership development and to focus on the development of compassionate and inclusive cultures that value the diversity in health workforces [36]. The underpinning evidence base highlighted the need to recognise that mainstream approaches to leadership development often take a leader-centric approach that fails to address dominant power relationships and perpetuates the status quo [36]; this resonates with some of the above discussion in this field about the power of dominant voices. This indicated a difference in both emphasis and approach; a move towards a blend of educational input that sought to address issues around identity, whilst also questioning which voices had been traditionally heard, and which less so. This blended approach, similar to many of the NHSLA leadership development programmes, incorporates a key focus on experiential group work creating safe spaces for its participants. These learning groups aim to enable participants to explore their leadership development in the context of systemic and individual challenges around power and identity. A 2019 evaluation of the Nye Bevan Programme [37], which is targeted towards aspiring or newly appointed board-level leaders noted an increased confidence and willingness to improve leadership practice around inclusion, and working with new, sometimes uncomfortably new, knowledge. Furthermore, the in-depth evaluation of the impact of the whole suite of programmes, specifically from a perspective of improving insights into cultural competence and its practice at individual, team and system levels could be an area of interest for future studies.

\section{Discussion}

The set of leadership development initiatives from the NHS Leadership Academy clearly focus on attitudes and behaviours as much as on skills and knowledge. It is not the intention of this paper to discuss or evaluate the programme curriculum at a detailed level-while some aspects of the curriculum are of interest at a micro-level, the focus here is more on what can be identified as the strategic method and goal. Taken as a whole, it can be determined that what is attempted is not so much a single or even a set of 'training' interventions but holistic cultural change at a system level. Developing a new theoretical model is not the primary purpose of this paper; rather, we seek to apply existing thinking and concepts to the specific and distinct approach towards cultural competence in the curriculum for existing and aspiring healthcare leaders espoused by the NHSLA. In doing so, questions arise not only about how cultural competence, cultural sensitivity, cultural humility or cultural intelligence are understood, processed and then re-expressed, but also about areas for potential study that might further enhance this evolving and nuanced concept. This forms the basis of the following commentary.

Turning firstly to the nomenclature, the language used by the NHSLA concentrates on 'equality, diversity and inclusion' (EDI), and the phrase 'cultural competence' features nowhere in the publicly available literature. However, what is expressed is congruent with what the literature would consider to be an approach towards cultural competence, with the work of Horvat et al. [3] (p. 21) again instructive here, given that "the diversity of approaches to cultural competence education reflected in the included studies, in terms of 
the terminology, goals of the interventions and descriptions of participants, supports our earlier description of the complexity of issues inherent within the field of cultural competence itself". Scholarly discussions have been exploring, testing and probing meanings for some time, and the concepts of cultural competence, cultural humility, cultural sensitivity and cultural intelligence, while intertwined, represent distinct strands of the phenomena. From the evidence examined in the case of NHSLA, there is little to indicate that these issues are demarcated in any distinguishable manner, accepting that "... uncertainty exists about the best and most effective way to educate health professionals in cultural competence" [3] (p. 23), and this is something that could be worthy of attention from the NHSLA when making curriculum revisions.

The strategic approach of the NHSLA finds resonance with the notion that the development of cultural competence does not necessarily need to be labelled as such to engender the outcome; the creation of a developmental space that is carefully designed to be rooted in a set of values or ethics can be productive in itself $[18,38]$. It has been noted that there has been, and remains, uncertainty about the most effective educational interventions for healthcare professionals around cultural competence [3], and that cultural competence education programmes would benefit from being more focussed and explicit about "their conceptual rationale, actual content, delivery... and approach to evaluation" (p. 23). Similarly, the attempt to embed EDI within the infrastructure of the curriculum is congruent with the 'active design' that much literature suggests is the hallmark of a culturally competent system [2,39]. The fact that NHSLA programmes place emphasis and importance on both reflection and reflexivity to contribute to this space or environment is parallel with the idea of developmental processes, challenging but involving practitioners to explore their own biases, preferences and motivators [2]; intercultural sensitivity, for example, engages attitudes, perceptions, values and the motivation to adapt, and cultural intelligence engages the ability to adapt to different cultural paradigms, using not just knowledge but socially adaptive skills $[10,18,27,40]$. A critique, however, can be noted around risks or sensitivities-excellent and skilled facilitation is needed to create safe spaces for both the individual and group exploration of issues around power, equality, inclusion and cultural competence. Arguably, a natural response to exposure to painful or challenging ideas and discussions is to use coping mechanisms, and this is where we move towards the interface between (curriculum) design and practice-no matter how culturally competent the design may be, at some point, it is the educator or facilitator who has to work with individuals and groups and help to create a safe space for discussion, exploration, and challenges. Again, the relationship between education and practice becomes prominent, as modelling cultural competence and comfort with discomfort is a critical step in helping those with whom educators work to do the same. It is also worth noting that the complexities around the issues at hand effectively assess what makes a culturally competent practitioner or leader difficult to achieve $[3,7,10,20]$; it is up to those who commission and design such programmes to make decisions regarding the relative importance of that. Clearly, knowledge can be meaningfully assessed, but it is harder to achieve that with regard to attitudes. In any case, it is impossible for any health worker to know all about the numerous cultural groups in almost any given society, given the multiple sources of diversity that prevail, and put plainly, "... simply having cultural knowledge and knowing about clients' culture is not sufficient to become a culturally competent healthcare practitioner" [18] (p. 600). A nuanced approach to cultural competence must, therefore, be about more than 'knowledge' or knowledge transfer, as can be observed in the programmes discussed here.

Common to all of the NHSLA programmes examined in this paper is the emphasis on applied learning, social group learning theory, and a focus on knowledge, skills and attitudes. This appears wholly congruent with the concepts of holistic education being anchored in something greater than knowledge, or even exposure to difference alone. Rooting development to a core set of values, rather than simply expressing these through learning objectives or outcomes, is given prominence in NHSLA programmes, and this again tends towards the focus being on attitudinal shifts leading to behaviour change, 
rather than giving primacy to knowledge inputs. Certainly, the approach of the NHSLA appears considerably closer to the culture and values end of the spectrum than that of 'fixing' a knowledge deficit; further, it can be inferred (but, we would argue, naturally follows) that the NHSLA approach is that knowledge of differences and cultures is not enough in itself to drive change, or at least not at a sufficient level or pace [13]. In other words, and put simply, 'teaching', including training, is not enough-there is a recognition and acknowledgement that there is a psycho-social, as well as pedagogic, dynamic at play and that this "is a lifelong process that is not accomplished through one workshop or crosscultural training" [11] (p. 43) [41]. So, the NHSLA does not, in its suite of the leadership programmes examined here, seek to teach or train 'cultural competence', but instead aims to diffuse the concepts through various—or possibly all, if a wide interpretation is applied to the concept of the leadership principles-aspects of its curriculum. The cerebral and the emotional are intertwined, recalling the argument of Kelly et al. [15] that cultural competence is not an event but a lifelong process that involves a circular and cyclical process of reflection, greater awareness, renewed reflection and so forth. Such work is never 'done', but with each cycle both cognitive knowledge and emotional intelligence will arguably deepen. Just as clinical practitioners willingly engage in ongoing professional development and a refreshing of knowledge and skills, so it can be argued that cultural competence in leadership development is not a destination but a process. Therefore, this becomes a circular, or perhaps more accurately, a triple-loop learning event-what was 'culturally competent' ten years ago may well not be nowadays, but if people and systems are equipped with the tools to utilise in response to a changing environment, sustainable change can occur. This, of course, also relates to one of the criticisms of this field of study and practice in more general terms, that 'this is never done', which is sometimes deployed as an argument not to start, or at least to stop at the point of discomfort. For educators, this is the antithesis of growth through developmental challenge.

What can this indicate about the two other features identified earlier in this paperthose of exposure to authentic (often service user) voices and the issue of mandating training? Certainly, patient centredness is a key concept in the NHSLA approach, although the extent to which this includes the expression and discussion of power, inclusion and difference is not known. As the literature has indicated, real-world encounters with diverse viewpoints, other cultures or simply with just differences are often considered important for developing the intellectual as well as emotional skills to explore cultural competence, stimulate intercultural curiosity, and develop cultural intelligence [2,39]. On one level, simply living or working in a multicultural environment can 'prime' curiosity about intercultural sensitivity and cultural intelligence [42], and the NHS is especially ethnically diverse. Whilst requiring further research, this is only a priming factor, however, and needs a well-designed pedagogy [43] to maximise learning potential. Lastly, the issue of mandating concepts around cultural competence (expressed by the NHSLA as equality, diversity and inclusion) is an interesting point; much of the literature is sceptical of the value of such an approach, with the risk of fostering resentment, tokenism or discomfort to a degree that learning is inhibited. There is little evidence of the NHSLA 'forcing' this through a curriculum route, but equally the fundamental leadership principles espoused by the NHSLA are not negotiable, and this could thus be interpreted as being mandatory. Further work would be beneficial here for exploring participant perceptions and experiences of the efficacy of what could be termed this quasi-mandatory approach.

Given all of the above, implications for practice and areas for further study can be proposed. Research into the development, delivery and impact of differing healthcare leadership development programmes composed of multi-disciplinary participants could provide insights into methods and efficacy. For example, is the exploration of intercultural sensitivity or the development of cultural intelligence easier to explore in multi-disciplinary groups of learners rather than uni-disciplinary ones? The evaluation and review of the leadership development curriculum for multi-professional and multi-disciplinary learning groups could also be profitable, especially if this includes a conscious discussion about 
the inclusion and expression of cultural competence, cultural sensitivity and cultural intelligence, which are all key leadership activities in contemporary and diverse healthcare environments. These would both add to the body of knowledge around cultural competence in the development of multi-disciplinary healthcare leaders.

\section{Conclusions}

In conclusion, having explored the phenomenon of how cultural competence is expressed in education and leadership development in healthcare, a number of points emerge. From a theoretical viewpoint, the concept of cultural competence can be located in a constructivist paradigm with an emphasis on critical reflection and reflexivity with a view to creating new knowledge [13]. Equipping those who work in healthcare-both clinical and, increasingly, non-clinical staff-with some notion of cultural competence clearly plays an existing and arguably increasing role in health services, and cultural competence interventions vary in their emphasis and methods. However, there is a clear and growing focus on what can be described as intercultural sensitivity and cultural intelligence, with a focus not just on knowledge but also on skills and attitudes [3,6]. Definition and meaning vary, perhaps understandably due to the need for authenticity in the field and the fact that authenticity will stem from highly local contingent factors. The literature illustrates evidence of a mixed, sometimes an eclectic mix, of approaches to develop cultural competence, on a spectrum including the simple provision of information, attempts to transfer knowledge either voluntarily or through mandatory means, through to curricula approaches that either include specific modular inputs or interventions, or at the other end of the spectrum, attempts to fundamentally design aspects of cultural competence into curriculum.

The manner in which the English National Health Service approaches cultural competence in the development of leaders was explored as a case study, given that the National Health Service Leadership Academy has been offering a wide variety of leadership development programmes for almost a decade. An examination of their (publicly available) information revealed that the focus is more on attitudes than on didactic knowledge transfer, and on strategic curriculum design rather than a specific method. Given the ethnic and racial diversity of the population of the United Kingdom, and that the NHS is a fully publicly funded health service, this emphasis on some form of aiming to develop a culturally competent workforce is, perhaps, reassuring. The focus on attitudinal shifts is interesting, as it speaks to the relative importance of behavioural change, which is a key feature of cultural intelligence. On one level, behavioural change (or indeed the self-management or regulation of behaviours) could be considered 'sufficient' in terms of health workers treating service users with dignity and respect, but many debates around institutional or systemic failings (in various spheres) identify attitudinal factors as key; eventually and at some level, attitudes and beliefs will always have an impact on behaviours. Intercultural literacy, for example, sits very much in the domain of knowledge, with members of the 'dominant' group learning about the norms of 'minority' groups. Is this part of cultural competence? Certainly. Is it sufficient? Almost certainly not. The NHSLA model approach towards equality, diversity and inclusion sought to move away from the 'knowledge input solves a deficit' approach, but these factors must remain part of the wider aims. It is this point where there is a recognition that cultural competence is in fact a phenomenon that needs to be considered or addressed but that it is all too easy for it to be labelled as 'too hard to do', not a priority given other issues, or that problems will resolve themselves over time. Cultural competence will bring us close to a tipping point where giving people the knowledge, language and space to explore the dimensions of cultural competence is critical, especially in healthcare. The experience of COVID-19, with a differential impact on groups in society in the context of existing (and sometimes entrenched) health inequalities has reinforced the need for health services at a strategic level, and by extension, the day to day practice of individuals involved in delivering healthcare to be culturally alert and competent. In that respect, the debate today can be framed as a morally and ethically 
imperative, rooted in value systems, and the implications and opportunities for those involved in the design and delivery of leadership development are rich.

Author Contributions: Conceptualization, S.G.; methodology, S.G.; software, not applicable; validation, S.G.; formal analysis, S.G.; investigation, S.G. and C.W.; resources, not applicable; data curation, not applicable; writing - original draft preparation, S.G.; writing-review and editing, S.G., C.W.; visualization, S.G.; supervision, not applicable; project administration, not applicable; funding acquisition, not applicable. All authors have read and agreed to the published version of the manuscript.

Funding: This research received no external funding.

Institutional Review Board Statement: Not applicable.

Informed Consent Statement: Not applicable.

Acknowledgments: The assistance of the Knowledge and Evidence Services staff at the Health Services Management Centre, University of Birmingham, in sourcing literature for the authors is gratefully acknowledged.

Conflicts of Interest: The authors declare no conflict of interest.

\section{References}

1. Guillaume, Y.R.F.; Dawson, J.F.; Woods, S.A.; Sacramenton, C.A.; West, M.A. Getting diversity at work to work: What we know and what we still don't know. J. Occup. Organ. Psychol. 2013, 6, 123-141. [CrossRef]

2. Bhui, K.; Warfa, N.; Edonya, P.; McKenzie, K.; Bhugra, D. Cultural competence in mental health care-A review of model evaluations. BMC Health Serv. Res. 2007, 7, 15. [CrossRef]

3. Horvat, L.; Horey, D.; Romios, P.; Kis-Rigo, J. Cultural competence education for health professionals. Cochrane Database Syst. Rev. 2014, 5, CD009405. [CrossRef] [PubMed]

4. blog.bham.ac.uk. Available online: https://blog.bham.ac.uk/socialsciencesbirmingham/2020/06/29/culturally-competentwhat-does-the-pandemic-tell-us / (accessed on 10 December 2021).

5. www.hee.nhs.uk. Available online: https://www.hee.nhs.uk/our-work/future-doctor (accessed on 10 December 2021).

6. Beach, M.C.; Price, E.G.; Gary, T.L.; Robinson, K.A.; Gozu, A.; Palacio, A.; Smarth, C.; Jenckes, M.W.; Feuerstein, C.; Bass, E.B.; et al. Cultural Competency: A Systematic Review of Health Care Provider Educational Interventions. Med. Care 2005, $43,356-373$. [CrossRef] [PubMed]

7. Anand, R.; Lahiri, I. Intercultural competence in health care: Developing skills for interculturally competent care. In The SAGE Handbook of Intercultural Competence; Deardorff, D.K., Ed.; SAGE Publications: Thousand Oaks, CA, USA, 2009 ; pp. $387-402$.

8. Luquis, R.R. Integrating the concept of cultural intelligence into health education and health promotion. Health Educ. J. 2021, 80, 833-843. [CrossRef]

9. Gunther, C.B. Addressing Unconscious Bias, Power, and Privilege to Increase Cultural Competence Skills in Healthcare Faculty: Intersecting Critical Race Theory and the Pyramid Model for Inter cultural Competence. Ph.D. Dissertation, University of South Carolina, Columbia, SC, USA, 2020. Available online: https:/ /scholarcommons.sc.edu/etd/6096 (accessed on 20 December 2021).

10. Armstrong, J.P. Assessing intercultural competence in international leadership courses: Developing the global leader. J. Leadersh. Educ. 2020, 19. [CrossRef]

11. Deardorff, D.K. Exploring the Significance of Culture in Leadership. New Dir. Stud. Leadersh. 2018, 160, 41-51. [CrossRef]

12. Northouse, P.G. Leadership: Theory and Practice, 8th ed.; SAGE: Thousand Oaks, CA, USA, 2018.

13. Garneau, A.B.; Pepin, J. Cultural Competence: A Constructivist Definition. J. Transcult. Nurs. 2015, 26, 9-15. [CrossRef]

14. Örtenblad, A.; Trehan, K.; Putnam, L.L. (Eds.) Exploring Morgan's Metaphors: Theory, Research, and Practice in Organizational Studies; SAGE Publications: Thousand Oaks, CA, USA, 2017.

15. Kelly, F.; Papadopoulos, I. Enhancing the cultural competence of healthcare professionals through an online course. Divers. Health Care 2009, 6, 77-84.

16. Markey, K.; Okantey, C. Nurturing cultural competence in nurse education through a values-based learning approach. Nurse Educ. Pract. 2019, 38, 153-156. [CrossRef]

17. Stathi, S.; Crisp, R.J. Intergroup contact and the projection of positivity. Int. J. Intercult. Relat. 2010, 34, 580-591. [CrossRef]

18. Henderson, S.; Horne, M.; Hills, R.; Kendall, E. Cultural competence in healthcare in the community: A concept analysis. Health Soc. Care Community 2018, 26, 590-603. [CrossRef] [PubMed]

19. Soule, I. Cultural Competence in Healthcare, An Emerging Theory. Adv. Nurs. Sci. 2014, 37, 48-60. [CrossRef] [PubMed]

20. Watt, K.; Abbott, A.; Reath, J. Developing cultural competence in general practitioners: An integrative review of the literature. BMC Fam. Pract. 2016, 17, 158. [CrossRef] [PubMed]

21. Sorensen, J.; Norredam, M.; Suurmond, J.; Carter-Pokras, O.; Garcia-Ramirez, M.; Krasnik, A. Need for ensuring cultural competence in medical programmes of European universities. BMC Med. Educ. 2019, 19, 21. [CrossRef] 
22. Pecukonis, E.; Doyle, O.; Bliss, D.L. Reducing barriers to interprofessional training: Promoting interprofessional cultural competence. J. Interprof. Care 2008, 22, 417-428. [CrossRef]

23. Holland, K.; Hogg, C. Cultural Awareness in Nursing and Health Care; Hodder Arnold: London, UK, 2010.

24. Papadopoulos, I.; Tilki, M.; Taylor, G. Transcultural Care: Issues in for Health Professionals; Quay Books: Wilts, UK, 1998.

25. Kripalani, S.; Bussey-Jones, J.; Katz, M.G. A prescription for cultural competence in medical education. J. Gen. Intern. Med. 2006, 21, 1116-1120. [CrossRef]

26. Seeleman, C.; Essink-Bot, M.L.; Stronks, K.; Ingleby, D. How should health service organizations respond to diversity? A content analysis of six approaches. BMC Health Serv. Res. 2015, 15, 510. [CrossRef]

27. Wang, C.; Shakespeare-Finch, J.; Dunne, M.P.; Hou, X.Y.; Khawaja, N.G. How much can our universities do in the development of cultural intelligence? A cross-sectional study among health care students. Nurse Educ. Today 2021, 103, 104956. [CrossRef]

28. Kai, J.; Bridgewater, R.; Spencer, J. “ "Just think of TB and Asians", that's all I ever hear': Medical learners' views about training to work in an ethnically diverse society. Med. Educ. 2001, 35, 250-256. [CrossRef]

29. Stone, J.R. Healthcare inequality, cross- cultural training, and bioethics: Principles and applications. Camb. Q. Healthc. Ethics 2008, 17, 216-226. [CrossRef]

30. Hunter, W.J. Cultural Competency in Health Care Providers' Ethical Decision Making and Moral Reasoning: Implications for Reducing Racial and Ethical Health Disparities for Diverse Populations. Ph.D. Thesis, Nova Southeastern University, UMI, Fort Lauderdale, FL, USA, 2008.

31. Available online: https://blogs.bmj.com/bmj/2018/01/22/stephane-mshepherd-cultural-awareness-training-for-health-professionalscan-haveunintended-consequences/ (accessed on 3 February 2022).

32. Lee, S.; Collins, F.L.; Simon-Kumar, R. Healthy Diversity? The Politics of Managing Emotions in an Ethnically Diverse Hospital Workforce. J. Intercult. Stud. 2020, 41, 389-404. [CrossRef]

33. Report of the Mid Staffordshire NHS Foundation Trust Public Inquiry: Executive Summary. 2013. Available online: http: //www.midstaffspublicinquiry.com/sites/default/files/report/Executive\%20summary.pdf (accessed on 12 December 2021).

34. www.efdmglobal.org. Available online: https://efmdglobal.org/wp-content/uploads/NHS_Leadership-Academy-Alliance_ Manchester_Business-EiP2016-Gold-FullCase-compressed.pdf (accessed on 14 December 2021).

35. www.leadershipacademy.nhs.uk. Available online: https://www.leadershipacademy.nhs.uk/resources/inclusion-equality-anddiversity / (accessed on 14 December 2021).

36. www.leadershipacademy.nhs.uk. Available online: https://www.leadershipacademy.nhs.uk/wp-content/uploads/2019/02/ Building-Leadership-for-Inclusion-Narrative.pdf (accessed on 16 December 2021).

37. www.leadershipacademy.nhs.uk. Available online: https://www.leadershipacademy.nhs.uk/resources/evaluation-of-ourprogrammes / (accessed on 16 December 2021).

38. Calvillo, E.; Clark, L.; Ballantyne, J.E.; Pacquiao, D.; Purnell, L.D.; Villarruel, A.M. Cultural competency in baccalaureate nursing education. J. Transcult. Nurs. 2009, 20, 137-145. [CrossRef] [PubMed]

39. Unver, V.; Uslu, Y.; Kocatepe, V.; Kuguoglu, S. Evaluation of cultural sensitivity in healthcare service among nursing students. Eur. J. Educ. Res. 2019, 8, 257-265. [CrossRef]

40. Arli, S.K.; Bakan, A.B. An investigation of the relationship between intercultural sensitivity and compassion in nurses. Int. J. Intercult. Relat. 2018, 63, 38-42. [CrossRef]

41. Campinha-Bacote, J. The Process of Cultural Competence in the Delivery of Healthcare Services: A model of care. J. Transcult. Nurs. Off. J. Transcult. Nurs. Soc. 2002, 13, 181-201. [CrossRef] [PubMed]

42. Göla, I.; Erkinb, Ö. Association between cultural intelligence and cultural sensitivity in nursing students: A cross-sectional descriptive study. Collegian 2019, 26, 485-491. [CrossRef]

43. Alexander, R. Essays on Pedagogy, 1st ed.; Routledge: London, UK, 2008. 